\title{
Lymphatic filariasis in children: Clinical features, infection burdens and future prospects for elimination
}

\author{
RANGANATHA KRISHNA SHENOY ${ }^{1}$ and MOSES J. BOCKARIE ${ }^{2}$ \\ ${ }^{1}$ Filariasis Chemotherapy Unit, TD Medical College Hospital, Alappuzha 688 011, Kerala, India \\ ${ }^{2}$ Centre for Neglected Tropical Diseases, Liverpool School of Tropical Medicine, Pembroke Place, Liverpool, L3 5QA, UK
}

(Received 13 December 2010; revised 24 February and 21 fune 2011; accepted 21 Fune 2011; first published online 3 August 2011)

SUMMARY

Lymphatic filariasis (LF), a common parasitic infection in tropical countries, causes lymphoedema of limbs, hydrocele and acute attacks of dermato-lymphangio-adenitis. Recent advances in diagnosis have helped to recognize that LF infection is often acquired in childhood. Newly available diagnostic techniques like sensitive antigen and antibody assays, Doppler ultrasonography and lymphoscintigraphy have helped to understand the subclinical pathology caused by this infection, which was hitherto generally believed to be irreversible. Recent studies indicate that drugs used in the mass drug administration (MDA) programme under GPELF are capable of reversing the sub-clinical lymphatic damage in children and provide benefits other than interruption of transmission. Albendazole and ivermectin used in MDA are effective against soil-transmitted helminthic infections common in children in LF endemic areas. Thus MDA had other 'beyond LF' benefits in treated children including increased appetite, weight gain, greater learning ability and concentration, better school attendance and prevention of anaemia. MDA should no longer be viewed as a measure for interrupting transmission alone. Recent findings of reversibility of early lymphatic pathology in treated children indicate that both MDA and 'foothygiene' measures are effective strategies in preventing and managing morbidity. Programme managers should effectively utilize this information to strengthen their advocacy efforts to achieve high and sustainable coverage in MDA.

Key words: Lymphatic filariasis, children, subclinical pathology, lymphangiectasia, reversal of lymphatic damage, prevention, mass drug administration, foot-care.

\section{INTRODUCTION}

Lymphatic filariasis (LF) is a mosquito-borne parasitic infection caused by filarial nematodes invading the human lymphatic tissue. It is an important public health problem endemic in 81 tropical countries. Globally, $1 \cdot 3$ billion people are at risk of this infection and about 120 million people are estimated to be affected by this disease (WHO, 2010a). Among the debilitating vector-borne tropical diseases, LF is ranked as the second most common cause of permanent physical disability worldwide (Taylor et al. 2010). The most common causative agent of the disease is Wuchereria bancrofti $(90 \%)$ with, to a lesser extent, Brugia malayi $(<10 \%)$ and rarely Brugia timori. The various genera of mosquitoes transmitting these parasites in different geographical locations include Culex, Anopheles, Aedes or Mansonia (Bockarie et al. 2009). LF is still a major cause of disability and a significant impediment to socioeconomic development in large areas of Asia, Africa, Western Pacific and in certain regions of the Americas (WHO, 2010b).

* Corresponding author: Centre for Neglected Tropical Diseases, Liverpool School of Tropical Medicine, Pembroke Place, Liverpool, L3 5QA, UK. Phone: +44 (0)1517053343. Fax: +44(0)151 7053370. Mobile: +44 07595020694. E-mail: moses.bockarie@liv.ac.uk
The disease manifestations of LF include chronic lymphoedema and elephantiasis mainly involving the limbs or sometimes the genital region; also hydrocele and chyluria, along with acute attacks of recurrent dermato-lymphangio-adenitis (ADLA) due to bacterial infections. These acute attacks are responsible for the worsening of lymphoedema and progression to elephantiasis (Pani et al. 1995). These clinical manifestations affect 44 million people globally. Studies indicate that once established, the lymphatic pathology resulting in disease manifestations cannot be easily reversed by available treatment modalities (Freedman et al. 1994, 1995; Taylor et al. 2010). Thus LF is better prevented. The recent advances in the understanding of pathogenesis of LF, the biology of the parasite, and development of newer diagnostic tools and chemotherapeutic agents that are safe and effective have all contributed to new hope that this disease can be eradicated globally (Bockarie and Molyneux, 2009; Taylor et al. 2010). This Global Programme for Elimination of LF (GPELF) launched in 2000 , is based on a dual approach consisting of (a) interruption of transmission of LF infection by treating the entire 'at risk' population in endemic countries by annual single dose administration of a two-drug combination consisting of albendazole along with either diethyl carbamazine (DEC) or ivermectin for a period of 4-6 years and (b) alleviation 
of the disability in those who already have the disease, by meticulous limb hygiene measures to prevent secondary bacterial and fungal infections that cause acute attacks of ADLA (Dreyer et al. 1999; Seim et al. 1999; Ottesen, 2000; Gyapong et al. 2005). GPELF is a rapidly expanding global health programme, probably the biggest in the history of public health (WHO, 2010c). Among the 81 countries considered endemic for LF, 53 have started implementing MDA to interrupt transmission. More than $2 \cdot 8$ billion treatments were delivered to a cumulative targeted population of 845 million people during 2000 to 2009. Of the 53 countries that have implemented MDA, $37(70 \%)$ have completed the number of rounds of MDA thought to be adequate in most settings to interrupt transmission $(\geqslant 5)$ in some of their implementation units. According to WHO estimates (WHO, 2010c), the overall economic benefit of the programme during 2000-2007 is around US\$24 billion.

Many aspects of LF point to a baffling disease. In the initial stages of infection, when the live adult worms are present in the lymphatics and usually associated with microfilaraemia, the individual does not have any outward disease manifestation. There is a long interval between acquiring the infection and development of disease manifestations. This finding has important implications in the light of the recent observation that LF infection is acquired in childhood in many instances. These children develop clinical disease mostly in their later adult life (Witt and Ottesen, 2001; Shenoy, 2006). The knowledge that children are infected in early life is consequent to introduction of many newly available diagnostic techniques like sensitive antigen assays that provide diagnosis even in low density infections (Weil and Ramzy, 2007), demonstration of sub-clinical lymphatic pathology and detection of presence of live adult worms in lymphatics by Doppler ultrasonography (Dreyer et al. 1999; Shenoy et al. 2007b) and lymph vessel damage by lymphoscintigraphy (Shenoy et al. 2008).

In the light of these findings it is quite relevant to discuss how LF affects the health prospects of children and the benefits they would accrue by elimination of this chronic, disabling and depressing illness from the community. This paper provides an up-to-date review of various aspects of LF in children including prevention of this infection.

\section{LYMPHATIC FILARIASIS IN CHILDREN}

Over 250 million children are exposed to LF worldwide. In some endemic communities, up to $10 \%$ of children under the age of 15 years are infected (Bockarie et al. 2002). More than 100 million children were treated in 2009 by mass drug administration to eliminate LF (WHO, 2010a). The outward disease manifestations of LF are seen mostly in adult life, even though there have been occasional reports from LF endemic areas of occurrence of lymphoedema and ADLA attacks in children including hydrocele in boys of pubertal age (Pani et al. 1991; Ramaiah et al. 1996; Witt and Ottesen, 2001; Weil and Ramzy, 2007). In the past there were several reasons why the incidence of LF in the paediatric population did not attract much attention. The foremost among them is the natural history of this disease, the early stages of which are remarkably silent and the progression to later stages of clinical disease is phenomenally slow. It is well known that the early stage of infection is characterized by asymptomatic microfilaraemia, which may continue so for varying periods. These subjects do not have any overt manifestation of the disease even though they have microfilariae $(\mathrm{mf})$ in their blood, usually detected by blood examination at night. The routine stained, thick blood-smear examination was found to be not sensitive enough to detect all microfilaraemics when compared to the more recently available immunochromatography (ICT) card test for the detection of filarial antigen in bancroftian filariasis (Weil and Ramzy, 2007). This is specially so when the density of $\mathrm{mf}$ is low or when the infection is in an early stage where the adult worms have not started producing $\mathrm{mf}$. Even during this early asymptomatic phase, children are shown to have dilatation of the lymphatics along with presence of live adult worms by ultrasonography (Dreyer et al. 1999; Shenoy et al. 2007a) and lymph vessel dilation by lymphoscintigraphy (Shenoy et al. 2008). This sub-clinical lymphatic pathology is difficult to diagnose by external examination. For these reasons, in the past many of these early infections in children were missed. Moreover, many earlier epidemiological studies did not include children less than 5 years.

Certain epidemiological studies and case reports in the past and more so in recent times, tests for filarial antigenaemia and ultrasound examination of the lymphatics in children mentioned above have all indicated that LF may be more importantly a childhood infection (Witt and Ottesen, 2001). During the course of research activities in a Brugia malayi endemic area at the Filariasis Chemotherapy Unit in TD Medical College Hospital, Alappuzha, India it was observed that among the asymptomatic, mf-positive subjects screened $\sim 30 \%$ were aged $\leqslant 20$ years, while $16 \cdot 3 \%$ were $<10$ years. In a study on prevention of ADLA attacks in brugian filariasis, $32 \%$ of the adults who had lymphoedema of several years duration recalled during their interview, that their disease first manifested before they were 15 years of age (Suma et al. 2002).

\section{Factors influencing LF infection in children}

Many past studies have reported clustering of young individuals with $\mathrm{mf}$ in a given household. This has 
been attributed to several mechanisms such as environmental factors facilitating exposure to infective mosquito bites (Vanamail et al. 1989, 1992) intra-uterine sensitisation due to maternal infection, higher prevalence of $\mathrm{mf}$ in children born to microfilaraemic mothers (Lammie et al. 1991) and parental infection (maternal, paternal or both) predisposing to higher prevalence of $\mathrm{mf}$ in the offspring (Das et al. 1997). Exposure to infection within the household appeared to be the most important cause for acquiring LF infection in childhood, more than prenatal sensitisation or genetic factors (Das et al. 1997).

\section{Clinical features of LF disease in childhood}

One well known clinical presentation of LF disease in the paediatric group is non-specific lymph node enlargement or soft tissue swelling in the inguinal region, axilla or neck. Biopsy and histopathological examination of such swellings had confirmed presence of dead adult filarial worms or their remnants (Jungmann et al. 1991). Several studies from endemic areas for LF have recorded the presence of lymphoedema of limbs in children, the incidence being higher in older children (Fig. 1). Higher grades of oedema are not common since this depends upon the duration of the illness. Hydrocele of the scrotal sac is also described in boys of pubertal age or older. Acute attacks of ADLA were noted in children both with and without lymphoedema (Pani et al.1991; Ramaiah et al. 1996; Fox et al. 2005). Episodes of ADLA are characterized by acute onset pain, fever, redness and tenderness of affected limb along with other constitutional symptoms like vomiting. The draining lymph nodes are usually painful and tender. The chronic manifestations are seen less often in children $<10$ years. In a recent study in a Brugia malayi endemic region, out of 7934 children screened for filarial infection and disease four girls between 10-15 years old had grade II lymphoedema (Shenoy et al. $2007 a$ ). Occasionally they present with filarial lymphangitis caused by death of the adult worms in the lymphatics, either spontaneous or after treatment with the antifilarial drug DEC. In such situations dilated, inflamed and tender lymphatic channels stand out as cords ("string sign") seen and palpated either by the side of the chest or inguinal region (Shenoy et al. 1998). Rarely chyluria and tropical pulmonary eosinophilia are also reported in children (Witt and Ottesen, 2001).

\section{Microfilaraemia in children}

Studies in different endemic populations on $\mathrm{mf}$ prevalence have shown a constant relationship between childhood and adult infections. The childhood $\mathrm{mf}$ prevalence rates were $\sim 30 \%$ of adult prevalence for $<10$ year olds and $\sim 69 \%$ for $10-19$

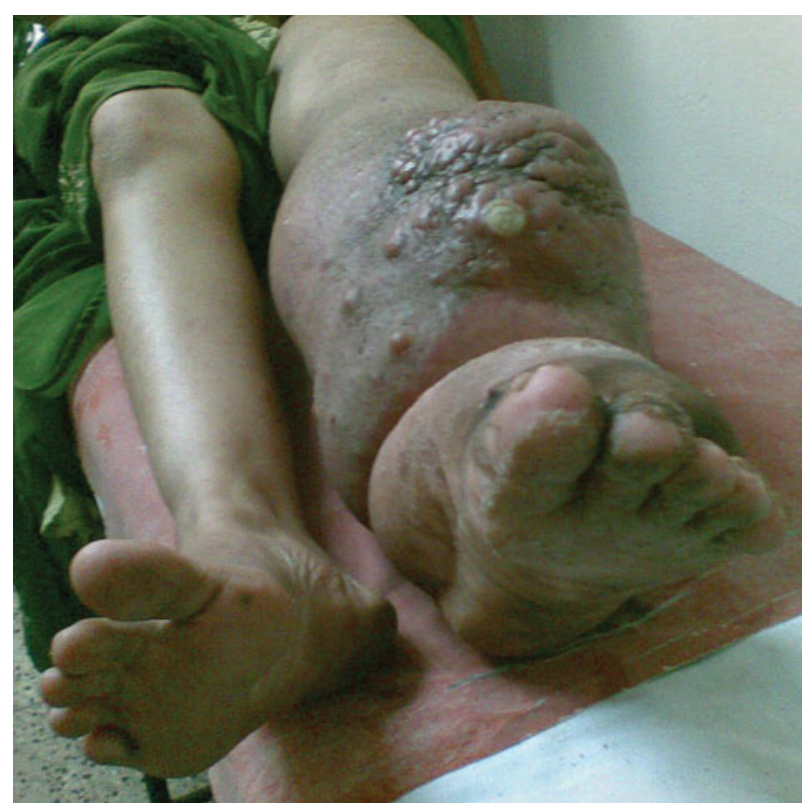

Fig. 1. Advanced lymphoedema in the left leg of a 14 year-old girl from an area endemic for lymphatic filariasis.

years olds (Witt and Ottesen, 2001). The mf counts tended to be lower in young children and were higher in older children. Offspring of microfilaraemic mothers had a higher risk of microfilaraemia when compared to those born of uninfected mothers (Alexander et al. 1998). In a study of Brugia malayi filariasis, 32 children were detected to have microfilaraemia ranging from 28 to $1812 / \mathrm{ml}$, three of them being just three years old (Shenoy et al. 2007a).

\section{Immuno-diagnosis of LF infection in childhood}

For bancroftian filariasis there are two sensitive tests presently available for detection of circulating filarial antigen, which can be carried out even during daytime in contrast to detection of $\mathrm{mf}$ by examination of blood at night. These are IC'T card test and $\mathrm{Og} 4 \mathrm{C} 3$ antibody based test in ELISA format (Weil and Ramzy, 2007). A positive test result indicates presence of live adult worms and thus active infection. The ICT card test is easier to perform than the ELISA test. Longitudinal studies conducted in children from endemic areas have demonstrated filarial antigenaemia in $6 \%$ of the two-year olds and the prevalence increased to $30 \%$ in four-year olds (Lammie et al. 1998). Test for the adult worm antigen is definitely a more sensitive method than blood $\mathrm{mf}$ counts to detect LF prevalence in children. If only the $\mathrm{mf}$ detection method by night blood examination is used for diagnosis, at least one-third of all infections would be missed (Witt and Ottesen, 2001). The filarial antigen prevalence (AGP) rates are always higher than the mf prevalence (MFP) in many field studies. One such study reported that AGP seen 
on ICT test was higher than MFP varying between $11 \cdot 3$ to $12 \%$ (Sunish et al. 2002). In another study, while $62 \%$ children had microfilaraemia, $81 \%$ had antigenaemia on Og4C3 testing (Fox et al. 2005).

Such antigen detection tests as described above are not available so far for a specific diagnosis of Brugia malayi infection. But a filaria-specific $\operatorname{IgG} 4$ antibody test using a recombinant antigen $(B m R 1)$ has been developed, which has 95-97\% sensitivity, 99\% specificity, 97\% positive predictive value and 99\% negative predictive value (Rahmah et al. 2001, 2003). This test is available in an ELISA format, as a dipstick test termed 'Brugia Rapid' (BR) and also in cassette form. The BR test was used in a study of LF infection in children in a Brugia-endemic area, where 7934 children were screened in villages for filarial infection and disease. In this population, it was found on screening that, while the prevalence of microfilaraemia by blood-smear examination was only $0 \cdot 38 \%$, the BR test was positive in $10 \cdot 97 \%$. Similar to what is seen by ICT card testing in bancroftian filariasis, the BR test thus identified nearly 27 times more 'infected' individuals in Brugia malayi infection than the conventional $\mathrm{mf}$ detection by blood smear examination (Shenoy et al. 2007a, 2009). From the above group, 100 children were enrolled for the chemotherapy studies, out of which 77 tested positive for filaria-specific IgG4 antibody. This IgG4 positive group had more individuals who had other unmistakable evidence of filarial infection or subclinical lymphatic pathology. Thus it was seen that $94 \%$ of the microfilaraemics, $86 \%$ of the children with live adult worm 'nests' detected on Doppler ultrasonography and $78 \%$ of the children who had abnormal lymphatics on lymphoscintigraphy (LSG) were IgG4 positive. Based on these observations it was concluded that a positive $\mathrm{BR}$ test is a reliable marker of B. malayi infection (Shenoy et al. 2009).

\section{Doppler and ultrasonography in LF infection in children}

One of the important recent tools in the diagnosis of LF infection is ultrasonography (USG) and Doppler sonography (DSG) (Shenoy et al. 2007a). Live adult worm nests, hydrocele, chylocele and lymph vessel dilatation (lymphangiectasia) can be detected by this procedure. As with adult males, older children with asymptomatic bancroftian microfilaraemia were shown to harbour live adult worms in the scrotal lymphatics. Ultrasound examination using a $7 \cdot 5 \mathrm{MHz}$ probe in eight boys aged 14-16 years revealed the existence of adult filarial worms indicated by the 'filaria dance sign' (FDS) in the 'nests' along with diffuse dilatation of the lymphatic vessels. FDS was also present in crural lymphatics and axillary lymph node in two girls aged 9 and 7 years respectively. FDS was detected even in one amicrofilaraemic child (Dreyer et al. 1999). USG is not as sensitive as $\mathrm{mf}$ detection on 'night' blood examination or tests for antigenaemia. Thus one study of $W$. bancrofti (WB) LF in 5-13 year old children found that while microfilaraemia was present in $62 \%$ and antigenaemia in $81 \%$, FDS was seen only in $15 \%$ (Fox et al. 2005).

In Brugia malayi infection where there is no genital involvement, one study using ultrasonography with a $7 \cdot 5 \mathrm{MHz}$ probe failed to locate any adult worm nests in the scrotal lymphatics in adults. It was concluded that this failure to demonstrate B. malayi (BM) parasites was due to either technological inadequacies (resolution of the probe, lack of Doppler techniques) or possibly due to intrinsic biological characteristics of the worm such as their smaller size, ability to locate to deeper tissues or inability to populate classical bancroftian locations such as the scrotal lymphatics (Shenoy et al. 2000). Thus in the recent study of BM infection in children, Colour Power Doppler (CPD) and Pulse Wave Doppler (PWD) examination were employed to locate adult worm nests, if any were present. This additional facility is essential to locate with confidence the small adult worm nests in BM infection and differentiate them from the echoes from blood vessels (Mand et al. 2006). By this method 18 nests with FDS were identified in 14 children comprising 11 boys and 3 girls. Four subjects had two 'nests' each. The youngest two children who showed FDS were only 3 years old. FDS was seen in the axilla in 12 children, in the thigh in two and in the epitrochlear and popliteal area in one child each (Fig. 2A and 2B). The worm nests were distinctly smaller and the wriggling movements were less rapid and less conspicuous than those of bancroftian filariasis. Lymph vessel dilations were observed on DSG in 38 out of the 100 enrolled children (Shenoy et al. 2007a).

\section{Lymphoscintigraphy in LF infection in children}

Lymphoscintigraphy has been carried out earlier in subjects with established lymphoedema of the limbs to delineate the underlying pathology of the lymph vessels and lymph nodes such as delayed or absent transport of the radiotracer, tortuous and bizarre deep lymphatics, dermal diffusion, retrograde tracer flow and faint or absent regional nodal visualization (Witte et al. 1993). More recently, lymphoscintigraphic studies have documented abnormal lymphatics even in asymptomatic individuals who had only circulating microfilaraemia due to $W$. bancrofti infection suggesting that lymphatic damage preceded onset of clinical disease (Freedman et al. 1994).

In a B. malayi-endemic area LSG was carried out in 100 children, 3-15 year-olds, who had either asymptomatic microfilaraemia, history or evidence of filarial disease or were positive for filariasis-specific 
A

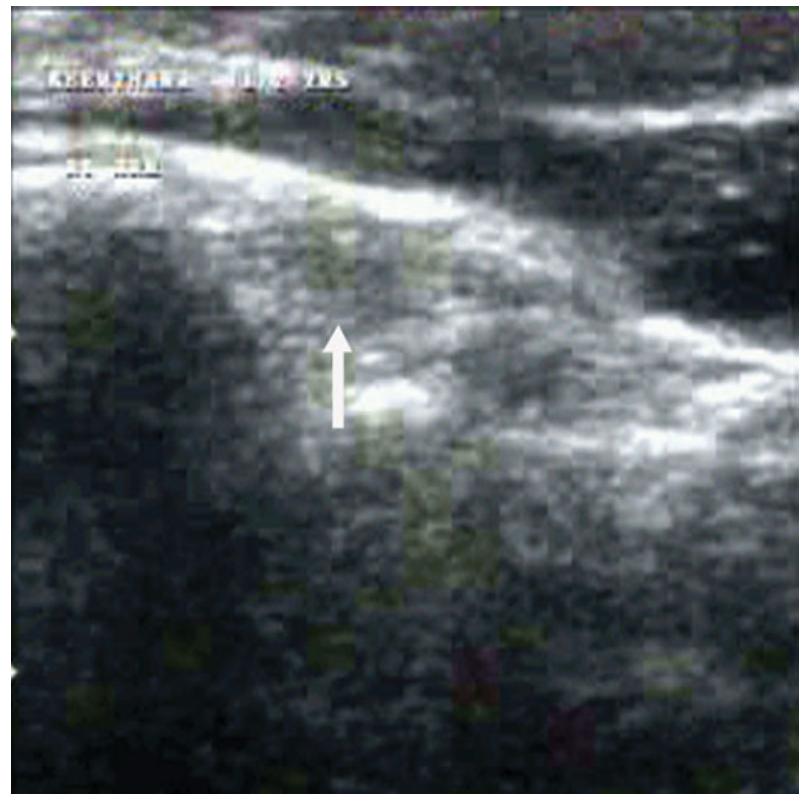

B

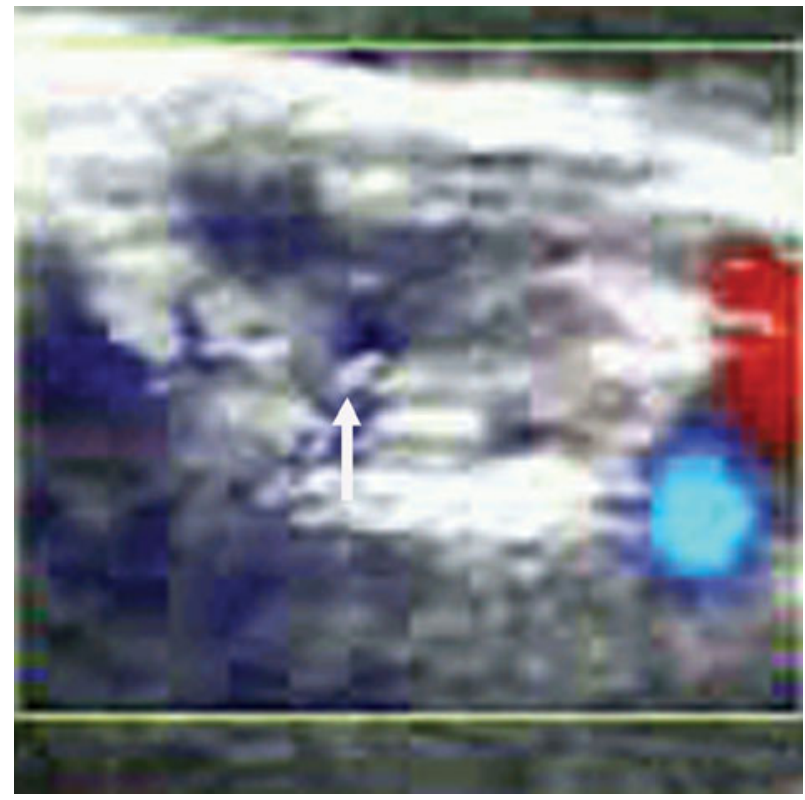

Fig. 2. (A). Ultrasound examination (B mode) of the right axilla of a 9 year-old male with asymptomatic microfilaremia showing the site of a dilated lymph vessel (arrow) with the 'filaria dance sign' (FDS), indicating presence of adult parasite 'nest'. (B). Colour Power Doppler (CPD) examination of the site of FDS above. Arrow points to the area of FDS, which shows irregular mosaic signals on CPD that are different from the characteristic signals produced from blood vessels.

IgG4 antibody. This was done by intradermally injecting $0.5 \mathrm{ml}$ of a solution containing $2 \mu \mathrm{Ci}$ of $\mathrm{Tc}^{99 \mathrm{~m}}$-tagged sulphur colloid into the first web spaces of both feet and hands and the lymphatics were imaged using gamma camera (Shenoy et al. 2007b). Dilated lymphatic channels were seen in 80 of the 100 children. This included $81 \%$ of the microfilaraemics (Fig. 3A and 3B), 79\% children with filarial disease and $79 \%$ of those who were only IgG4 positive. Five children who had lymph vessel dilation were only three years old. Dilated lymph vessels $(\mathrm{LV})$, with tortuosity in some subjects, were commonly seen in the lower limbs. Upper limb involvement was less common. Both in the upper and lower limbs the entire length of the affected lymphatics appeared dilated. Inguinal, axillary and less commonly popliteal or epitrochlear lymph nodes were visualized on lymphoscintigraphy in all the 100 children examined. This study demonstrated that LF infection in children is associated with subclinical pathology even in the pre symptomatic phase of the infection when they had only microfilaraemia or were only IgG4 positive (Shenoy et al. 2008).

\section{Histopathology of LF infection in children}

A not uncommon clinical manifestation of bancroftian filariasis in children in endemic areas is presentation with enlarged lymph nodes or soft tissue swellings in the axilla, groin or neck. Biopsy and histopathological studies of these lymph nodes and tissues demonstrated the presence of adult filarial parasites and $\mathrm{mf}$ associated with marked dilation of lymph vessels in these children who did not have any other evidence of filarial disease (Figueredo-Silva et al. 1994; Dreyer et al. 2001). The fact that lymphangiectasia is not restricted to the exact segment where the adult worms are located indicates that it may be the result of the action of soluble parasite-specific factors or excretory products on responsive lymphatic endothelial cells. Live parasites were not associated with any inflammatory reaction in the lymph vessels, while dead worms induced granulomatous inflammatory reaction with variable numbers of eosinophils, lymphocytes, plasma cells and large macrophages. There may be large numbers of eosinophils, especially in cases treated with DEC (Figueredo-Silva et al. 2002).

Another important aspect of the pathology of LF has been the finding that the bacterial endosymbionts Wolbachia, present in the filarial parasites, may be responsible for the inflammatory process associated with the progression of this disease. Exposure to Wolbachia lipoproteins could be responsible for promoting lymphangiogenesis, lymphatic endothelial proliferation and dilation of lymph vessels, which are known to result in chronic manifestations of LF disease ('Taylor et al. 2010). Studies are needed on this aspect of LF in children.

\section{Social and psychological impact of LF in the young}

LF disease poses several important problems in childhood and adolescence. The social and 
A

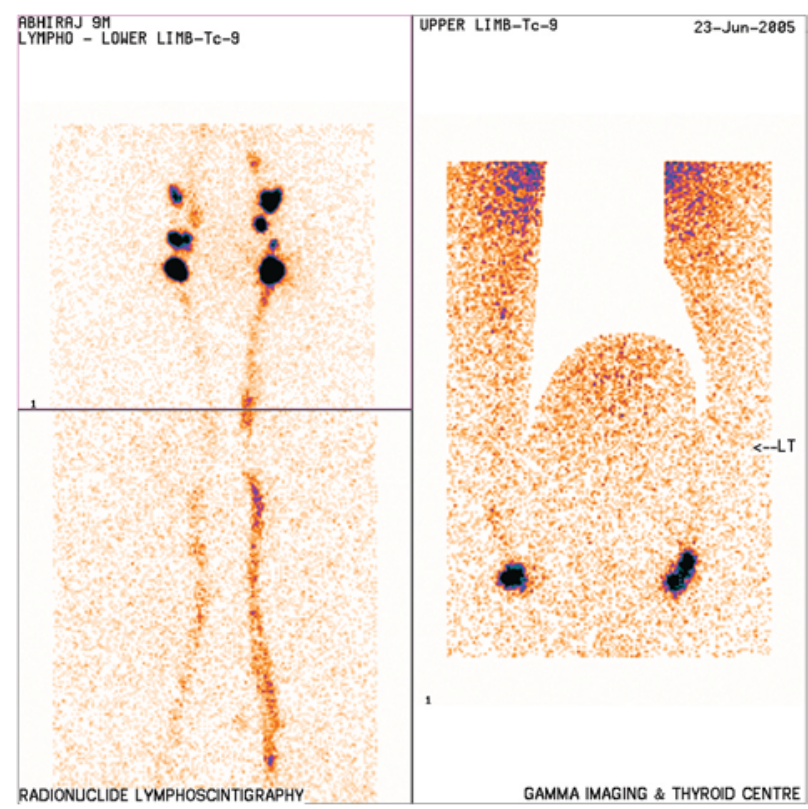

B

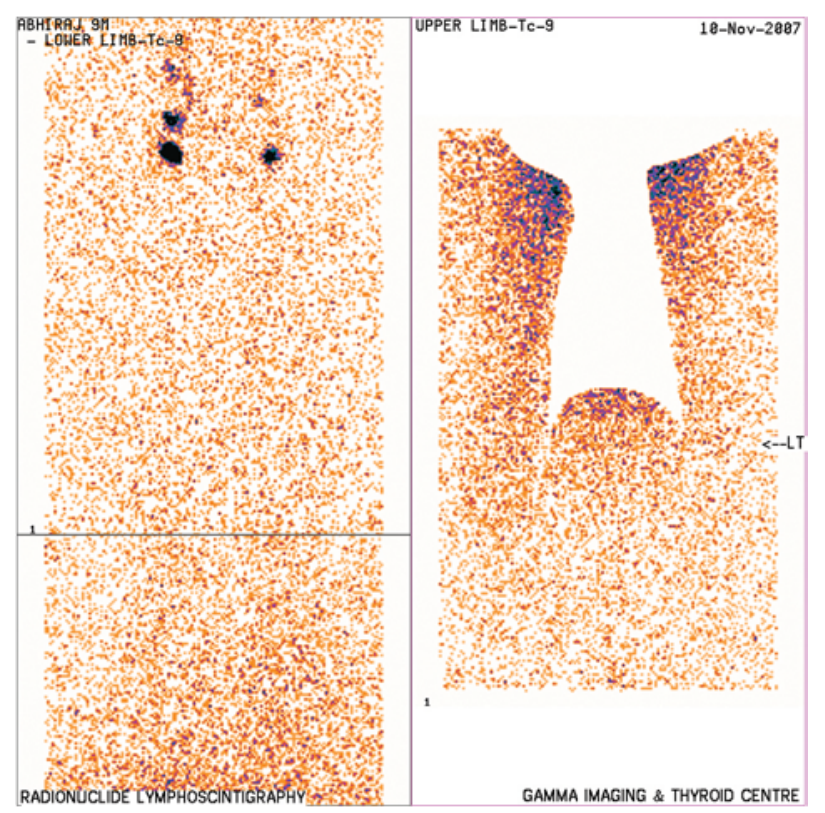

Fig. 3. (A). Lymphoscintigraph of a 9 year-old boy with asymptomatic microfilaraemia showing lymph vessel dilation in both legs (left panel). (B). Note absence of lymph vessel dilation in the legs on 30 months follow up, after administration of five doses of DEC and albendazole once every six months.

psychological impact of this malady in this age group had been addressed in some studies in the affected communities. The clinical manifestations of LF in the younger age group were associated with social stigma and psychological problems that interfered with their education and self-confidence. In a study conducted in two villages in South India, 28 subjects aged $<20$ years had clinical manifestations that included lymphoedema and ADLA attacks. Hydrocele was present in boys aged 10-15 years. Their social problems included feeling of shame, embarrassment and ridicule especially due to hydrocele. The presence of hydrocele and lymphoedema even interfered with their conventional school dress. Frequent absence from school due to the acute attacks and sometimes discontinuation of studies in this population would have socio-economic implications for their future (Ramaiah and Vijay Kumar, 2000). This chronic disease may thus interfere with the quality of life of these children as they develop.

\section{Diagnosis of LF infection and disease in children}

Children living in LF-endemic communities have a higher risk of developing the infection, which is first acquired by many in their early childhood itself. The dilatation of the lymphatics, which is the early and basic sub-clinical pathology induced by the adult worms, once established is reported to be irreversible even with treatment (Freedman et al. 1995). In the affected limbs this persistent lymphatic damage predisposes to later development of lymph stasis, secondary bacterial infections precipitating ADLA attacks, consequent development of lymphoedema and its progression (Shenoy et al. 1999). Therefore detection of LF infection in the early asymptomatic stage itself and prompt treatment are important in preventing future chronic disability. The investigations that are useful for diagnosis in the early stages are examination of thick blood-smears obtained at night for presence of $\mathrm{mf}$; Nuclepore membrane filtration to quantify the parasitaemia, ICT card test or $\mathrm{Og} 4 \mathrm{C} 3$ test for filarial antigenaemia in $\mathrm{WB}$ infection and filariasis-specific IgG4 antibody test in BM infection. USG and DSG may be employed in doubtful situations to detect any FDS, though this is not as specific as the other two methods. LSG being a costly investigation is rarely employed for routine diagnosis of LF infection. There is scope for future development of diagnostics in LF infection in children such as refinement in the use of PCR technique and detection of filariasis-specific antigen/ antibodies.

Once the subject presents with swelling of the limbs or with an ADLA attack, a clinical diagnosis of LF is suspected under certain circumstances such as the subject residing in a region endemic for $\mathrm{LF}$, familial clustering of such cases, swelling more often unilateral involving the lower limbs or rarely upper limbs of long duration except during initial stages and asymmetry of the swelling when bilateral. The oedema is pitting in early stages, though non-pitting due to thickening of skin in the later stages. This stage 
is uncommon in children since the thickening of skin depends on longer duration of disease. Presentation with lymphoedema or hydrocele are seen mostly in children $>10$ years of age.

In special situations, investigations like CT, MRI and MR angiography are sometimes employed in children to differentiate filarial swelling from other causes of lymphoedema like malformations of lymph vessels seen in association with certain congenital diseases (Stanton et al. 2000).

\section{Management of LF infection and disease in children}

Treatment of LF infection. DEC is the drug of choice when there is active infection from $W$. bancrofti, B. malayi or B. timori. Though it is very effective as a microfilaricidal agent, DEC kills only around $50 \%$ of adult worms. Even though the standard dose of DEC recommended in LF over the years was $6 \mathrm{mg} / \mathrm{kg}$ daily for 12 days, recent studies have shown that a single dose of $6 \mathrm{mg} / \mathrm{kg}$ is as effective as the 12-day course both against the $\mathrm{mf}$ and adult worms (Gyapong et al. 2005). In early stages of lymphoedema, DEC may be helpful if there is active filarial infection and its use might prevent further lymphatic damage through its macrofilaricidal action. The other antifilarial drugs, ivermectin and albendazole, even though important in the sustained reduction of blood $\mathrm{mf}$ levels and arresting LF transmission, have no role in the management of lymphoedema or acute attacks.

Treatment of ADLA. These attacks can easily be treated and further episodes prevented. Bed rest, elevation of the affected limb and symptomatic treatment with simple drugs like paracetamol are sufficient in mild cases. Any local precipitating 'entry lesions' like injury and bacterial or fungal infection should be treated with local antibiotic or antifungal ointments or with systemic antibiotics where needed. Proper and regular practice of 'local hygiene' measures of the affected limbs are effective in preventing ADLA attacks (Shenoy et al. 1998). This is aimed at prevention of fungal and bacterial infections and has become the mainstay for disability alleviation in GPELF (Ottesen, 2000).

Treatment of lymphoedema. In early lymphoedema, occasionally seen in older children, applying elastocrepe bandage to the affected limb while ambulant and keeping the limb elevated at night are effective to obtain relief.

Anti-Wolbachia therapy in LF. Studies in adults with LF infection have documented the encouraging results of treatment with doxycycline at $200 \mathrm{mg}$ daily given for varying periods from 3 to 8 weeks. This has resulted in long-term sterility and eventual death of the adult worms (Taylor et al. 2010). There are presently no reports of such a protracted course of doxycycline treatment in children with LF. Due to the adverse effects that doxycycline is known to cause, this drug is generally not used in pregnant women and children (Taylor et al. 2010). It may be recalled here that in a recent study on BM infection in children the presently available anti-filarial drugs are generally well tolerated and effective in clearing microfilaraemia and the FDS indicating presence of live adult worms and also in reversing the early subclinical lymphatic pathology (Shenoy et al. 2009).

\section{Prevention of LF infection and disease in children}

Prevention of filarial infection in children can be achieved either through arresting the parasite transmission by reducing the $\mathrm{mf}$ load in the community employing mass drug administration (MDA), by thorough vector control or by preventing man-vector contact. Of these, MDA initiated by GPELF is now the most effective method since 2000. Presently 53 out of the initially identified 81 LF-endemic countries are under MDA (WHO, 2010c).

In countries endemic for bancroftian infection, 4-6 annual rounds of MDA have been found to be effective in lowering the mf levels in the community, in reducing the filarial antigen levels in the population including children and thus preventing parasite transmission in the treated communities (Ramaiah et al. 2002, 2007). In children from a WBendemic area treated with DEC and albendazole, FDS indicating adult worm nests disappeared after treatment (Fox et al. 2005). In assessing the impact of GPELF after 8 years of its institution, it is reported that this has prevented LF disease in 6.6 million newborns who would otherwise have acquired LF, thus averting in their lifetimes nearly 1.4 million cases of hydrocele, 800,000 cases of lymphoedema and 4.4 million cases of subclinical disease (Ottesen et al. 2008).

In a recently published study in children with Brugia malayi infection in India, it was demonstrated for the first time that anti-filarial drugs in doses employed in MDA by the GPELF, reversed the subclinical lymphatic pathology. In this study, tests for antifilarial IgG4, Doppler ultrasonography and lymphoscintigraphy were employed to identify the sub-clinical pathology in 100 children. They were either asymptomatic microfilaraemic, amicrofilaraemic but had past or present filarial disease or were asymptomatic amicrofilaraemic but positive only for IgG4. They were treated every six months with single combined doses of DEC $(6 \mathrm{mg} / \mathrm{kg})$ and albendazole $(400 \mathrm{mg})$ and followed up for three years. At the end of three years all children were amicrofilaraemic, FDS was abolished in the 14 children who were initially positive and the lymphoedema (grade II) had 
disappeared in all the four girls who had it initially, Lymphoscintigraphy at the last follow-up revealed that the lymph node and vessel damage observed during enrollment had reversed in $89.6 \%$ children. They included $96 \%$ of the $\mathrm{mf}$ positives, $85 \%$ of those with filarial disease and $87 \cdot 5 \%$ of those who were only positive for IgG4 (Shenoy et al. 2009). These results suggest that MDA is effective in reversing the subclinical pathology induced by the adult worms in children, if they are treated early in LF infection. This is in contrast to the findings reported in adults that the sub-clinical pathology once established is irreversible even with treatment (Freedman et al. 1995).

In the context of GPELF, the present guidelines for interruption of LF transmission require that after achieving a microfilaraemia prevalence status of $<1 \%$, a sample of 3000 children in the age group of 6-10 years should be tested for filarial antigenaemia with the ICT card test. If one or more children are positive, MDA has to be continued and also that such children are investigated and treated (WHO, 2000). This indicates the importance of parasitaemia in children in the transmission of LF.

In the present scenario of integrated vector management, the use of mosquito repellant-impregnated bed nets will be helpful in preventing LF infection in children in communities where there is co-endemicity of malaria, filariasis and other vectorborne parasitic infections.

\section{DISCUSSION}

It is being increasingly appreciated that, in endemic countries, filarial parasites infect children early in life. Though asymptomatic at this stage, recently available diagnostic techniques have made it possible to recognize LF infection and sub-clinical lymphatic damage in children. Apart from microfilaraemia, circulating filarial antigen in WB infection, filariasisspecific IgG4 antibody in BM infection, 'nests' of live adult parasites in the lymphatics and the subclinical changes in the lymph vessels can all now be detected. The sub-clinical pathology caused by the adult worms in infected children is extensive in the affected lymph vessels, though these subjects do not have disease manifestations until later in their adult life (Figueredo-Silva et al. 2002; Shenoy et al. 2008). The gravity of this public health issue in LF-endemic countries can be understood when it is known that LF infection occurs in $10 \%$ of the 'at-risk' population; $66.7 \%$ of such infections have sub-clinical damage, $20 \cdot 8 \%$ result in hydrocele and $12 \cdot 5 \%$ result in lymphoedema (Ottesen et al. 2008).

The MDA under GPELF being carried out among the 'at-risk' population in endemic countries for the last nine years has prevented disease in many children born after MDA commenced (Ottesen et al. 2008). In this programme children aged 2 years and above are included. The drug albendazole, with either DEC or ivermectin, used in mass drug treatment is mainly aimed at preventing parasite transmission and ultimately eliminating LF infection in treated communities. The intention is thus primary prevention of the disease. In the last eight years, MDA is estimated to have prevented LF disease in 6.6 million future newborns and averted in their lifetimes nearly 1.4 million cases of hydrocele, 800,000 cases of lymphoedema and 4.4 million cases of subclinical disease which they would otherwise are expected to develop (Ottesen et al. 2008).

There have been many earlier reports indicating the beneficial effects of drugs used in MDA especially DEC, such as improvement or reduction in the incidence of lymphoedema, hydrocele and ADLA in treated communities (March et al. 1960; Meyrowitsch and Simonsen, 1998; Bockarie et al. 2002; Meyrowitsch et al. 2004). Hitherto the assumption has been that the underlying lymphangiectasia could not be reversed even after treatment. Now it has been shown that, at least in B. malayi infection in children, the sub-clinical pathology can be reversed by the drugs DEC and albendazole in doses used in MDA (Shenoy et al. 2007a). This indicates that MDA is also capable of secondary prevention of LF. This finding has tremendous impact in the present LF elimination campaign.

The 'foot care' or 'limb hygiene' programme recommended under disability management of GPELF is very effective in preventing the occurrence of acute ADLA attacks in LF. These episodes are responsible for worsening the lymphatic damage and future development of swelling in the limbs (Shenoy et al. 1999). In children having asymptomatic LF infection, the underlying sub-clinical lymphatic damage predisposes them to acute attacks following minor injuries or bacterial skin infections. They are thus prone to develop lymphoedema which, when established, is difficult to reverse. So to prevent this disability, regular 'foot-care' must be instituted in 'at-risk' children in endemic communities from an early age. Innovative approaches, such as introduction of this foot-care programme in the school curriculum in endemic areas, would be a progressive step in this direction (Shenoy, 2006).

Apart from elimination of LF infection, mass treatment of children in endemic countries with albendazole and ivermectin has been shown to have other 'beyond-LF' benefits. Both these drugs are also effective against intestinal, soil-transmitted helminthiases (STH), namely hookworm, roundworm and whipworm infection, which are very common in children. In addition, ivermectin is efficacious against Strongyloides stercoralis. Recent studies indicate that combining ivermectin and albendazole result in high cure rates in Trichuris trichura infection also (Knopp et al. 2010). STH infections are prevalent throughout the pan-tropical belt where LF is endemic (de Silva 
et al. 2003). Mass treatment with these drugs has beneficial effects in treated children including increased appetite and weight gain, greater learning ability and concentration, better school attendance and prevention of anaemia (Stephenson et al. 1993; Adams et al. 1994).

The above findings support the need for strengthening efforts to ensure high treatment coverage among eligible children 'at-risk' since the benefits extend well beyond clearance of LF infections. Programme managers and pediatricians in endemic countries recognizing the 'invisible disability' imposed by filarial infection should institute simple measures of hygiene to alleviate morbidity in these children. MDA should no longer be viewed as a measure for interrupting transmission alone. Recent findings of reversibility of early lymphatic pathology in treated children provide ample proof that both the MDA strategy and 'foot-hygiene' measures can be viewed as an effective measure in preventing and managing morbidity. Programme managers should effectively utilize this information to strengthen their advocacy efforts to achieve high and sustainable coverage in MDA (Shenoy et al. 2009).

\section{ACKNOWLEDGEMENTS}

We thank Rinki Deb for her assistance with the manuscript. The Centre for Neglected Tropical Diseases, Liverpool School of Tropical Medicine is funded by the UK Department for International Development (DFID). The British Society for Parasitology covered the local travel and accommodation expenses for Moses Bockarie.

\section{REFERENCES}

Adams, E. J., Stephenson, L. S., Latham, M. C. and Kinoti, S. N. (1994). Physical activity and growth of Kenyan school children with hookworm, Trichuris trichiura and Ascaris lumbricoides infections are improved after treatment with albendazole. Fournal of Nutrition 124, 1199-1206.

Alexander, N.D., Kazura, J.W., Bockarie, M. J., Perry, R. T., Dimber, Z. B., Grenfell, B. T. and Alpers, M.P. (1998). Parental infection confounded with local infection intensity as risk factors for childhood microfilaraemia in bancroftian filariasis. Transactions of the Royal Society of Tropical Medicine and Hygiene 92, 23-24.

Bockarie, M. J. and Molyneux, D.H. (2009). The end of lymphatic filariasis? British Medical Fournal 338, b1686.

Bockarie, M. J., Pedersen, E. M., White, G. B. and Michael, E. (2009). Role of vector control in the global program to eliminate lymphatic filariasis. Annual Review of Entomology 54, 469-487. doi: 10.1146/annurev. ento.54.110807.090626

Bockarie, M. J., Tisch, D. J., Kastens, W., Alexander, N. D., Dimber, Z., Bockarie, F., Ibam, E., Alpers, M. P. and Kazura, J. W. (2002). Mass treatment to eliminate filariasis in Papua New Guinea. New England Fournal of Medicine 347, 1841-1848. doi: 10.1056/NEJMoa021309 347/23/1841 [pii].

Das, P. K., Sirvidya, A., Vanamail, P., Ramaiah, K. D., Pani, S.P., Michael, E. and Bundy, D. A. (1997). Wuchereria bancrofti microfilaraemia in children in relation to parental infection status. Transactions of Royal Society of Tropical Medicine and Hygiene 91, 677-679.

de Silva, N. R., Brooker, S., Hotez, P. J., Montresor, A., Engels, D. and Savioli, L. (2003). Soil-transmitted helminth infections: updating the global picture. Trends in Parasitology 19, 547-551. doi: S1471492203002757 [pii].

Dreyer, G., Figueredo-Silva, J., Carvalho, K., Amaral, F. and Ottesen, E. A. (2001). Lymphatic filariasis in children: adenopathy and its evolution in two young girls. American Fournal of Tropical Medicine and Hygiene 65, 204-207.

Dreyer, G., Noroes, J., Addiss, D., Santos, A., Medeiros, Z. and Figueredo-Silva, J. (1999). Bancroftian filariasis in a paediatric population: an ultrasonographic study. Transactions of Royal Society of Tropical Medicine and Hygiene 93, 633-636.

Figueredo-Silva, J., Dreyer, G., Guimaraes, K., Brandt, C. and Medeiros, Z. (1994). Bancroftian lymphadenopathy: absence of eosinophils in tissues despite peripheral blood hypereosinophilia. Fournal of Tropical Medicine and Hygiene 97, 55-59.

Figueredo-Silva, J., Noroes, J., Cedenho, A. and Dreyer, G. (2002). The histopathology of bancroftian filariasis revisited: the role of the adult worm in the lymphatic-vessel disease. Annals of Tropical Medicine $\xi^{\circ}$ Parasitology 96, 531-541. doi: 10.1179/000349802125001348.

Fox, L. M., Furness, B. W., Haser, J. K., Brissau, J. M., LouisCharles, J., Wilson, S. F., Addiss, D. G., Lammie, P. J. and Beach, M. J. (2005). Ultrasonographic examination of Haitian children with lymphatic filariasis: a longitudinal assessment in the context of antifilarial drug treatment. American Fournal of Tropical Medicine and Hygiene 72, 642-648. doi: 72/5/642 [pii].

Freedman, D. O., Bui, T., De Almeida Filho, P. J., Braga, C., Maia e Silva, M. C., Maciel, A. and Furtado, A. F. (1995). Lymphoscintigraphic assessment of the effect of diethylcarbamazine treatment on lymphatic damage in human bancroftian filariasis. American fournal of Tropical Medicine and Hygiene 52, 258-261.

Freedman, D. O., de Almeida Filho, P.J., Besh, S., Maia e Silva, M. C., Braga, C. and Maciel, A. (1994). Lymphoscintigraphic analysis of lymphatic abnormalities in symptomatic and asymptomatic human filariasis. Fournal of Infectious Diseases 170, 927-933.

Gyapong, J. O., Kumaraswami, V., Biswas, G. and Ottesen, E. A. (2005). Treatment strategies underpinning the global programme to eliminate lymphatic filariasis. Expert Opinion Pharmacotherapy 6, 179200. doi: EOP060203 [pii] 10.1517/14656566.6.2.179.

Jungmann, P., Figueredo-Silva, J. and Dreyer, G. (1991). Bancroftian lymphadenopathy: a histopathologic study of fifty-eight cases from northeastern Brazil. American Fournal of Tropical Medicine and Hygiene 45, 325331.

Knopp, S., Mohammed, K. A., Speich, B., Hattendorf, J., Khamis, I. S., Stothard, J.R., Rollinson, D., Marti, H. and Utzinger, J. (2010). Albendazole and mebendazole administered alone or in combination with ivermectin against Trichuris trichiura: a randomized controlled trial. Clinical Infectious Diseases 51, 1420-1428.

Lammie, P. J., Hitch, W. L., Walker Allen, E. M., Hightower, W. and Eberhard, M.L. (1991). Maternal filarial infection as risk factor for infection in children. Lancet 337, 1005-1006.

Lammie, P. J., Reiss, M. D., Dimock, K. A., Streit, T. G., Roberts, J. M. and Eberhard, M. L. (1998). Longitudinal analysis of the development of filarial infection and antifilarial immunity in a cohort of Haitian children. American Fournal of Tropical Medicine and Hygiene 59, 217-221.

Mand, S., Supali, T., Djuardi, J., Kar, S., Ravindran, B. and Hoerauf, A. (2006). Detection of adult Brugia malayi filariae by ultrasonography in humans in India and Indonesia. Tropical Medicine and International Health 11, 1375-1381. doi: TMI1693 [pii]10.1111/j.13653156.2006.01693.x.

March, H. N., Laigret, J., Kessel, J. F. and Bambridge, B. (1960). Reduction in the prevalence of clinical filariasis in Tahiti following adoption of a control program. American Fournal of Tropical Medicine and Hygiene $\mathbf{9}$, 180-184.

Meyrowitsch, D. W. and Simonsen, P.E. (1998). Long-term effect of mass diethylcarbamazine chemotherapy on bancroftian filariasis, results at four years after start of treatment. Transactions of the Royal Society of Tropical Medicine and Hygiene 92, 98-103.

Meyrowitsch, D. W., Simonsen, P. E. and Magesa, S. M. (2004). Longterm effect of three different strategies for mass diethylcarbamazine administration in bancroftian filariasis: follow-up at 10 years after treatment. Transactions of the Royal Society of Tropical Medicine and Hygiene 98, 627-634. doi: 10.1016/j.trstmh.2004.01.004 S0035920304001300 [pii]. Ottesen, E. A. (2000). The global programme to eliminate lymphatic filariasis. Tropical Medicine and International Health 5, 591-594. doi: tmi620 [pii].

Ottesen, E. A., Hooper, P. J., Bradley, M. and Biswas, G. (2008). The global programme to eliminate lymphatic filariasis: health impact after 8 years. PLOS Neglected Tropical Diseases 2, e317. doi: 10.1371/journal. pntd.0000317.

Pani, S. P., Balakrishnan, N., Srividya, A., Bundy, D. A. and Grenfell, B. T. (1991). Clinical epidemiology of bancroftian filariasis: effect of age and gender. Transactions of Royal Society of Tropical Medicine and Hygiene 85, 260-264. 
Pani, S. P., Yuvaraj, J., Vanamail, P., Dhanda, V., Michael, E., Grenfell, B. T. and Bundy, D. A. (1995). Episodic adenolymphangitis and lymphoedema in patients with bancroftian filariasis. Transactions of Royal Society of Tropical Medicine and Hygiene 89, 72-74.

Ramaiah, K. D., Ramu, K., Kumar, K. N. and Guyatt, H. (1996). Epidemiology of acute filarial episodes caused by Wuchereria bancrofti infection in two rural villages in Tamil, Nadu, south India. Transactions of Royal Society of Tropical Medicine and Hygiene $\mathbf{9 0}$ 639-643.

Ramaiah, K. D., Vanamail, P. and Das, P. K. (2007). Changes in Wuchereria bancrofti infection in a highly endemic community following 10 rounds of mass administration of diethylcarbamazine. Transactions of Royal Society of Tropical Medicine and Hygiene 101, 250-255. doi: S0035-9203(06) 00196-9 [pii] 10.1016/j.trstmh.2006.05.007.

Ramaiah, K. D., Vanamail, P., Pani, S. P., Yuvaraj, J. and Das, P. K. (2002). The effect of six rounds of single dose mass treatment with diethylcarbamazine or ivermectin on Wuchereria bancrofti infection and its implications for lymphatic filariasis elimination. Tropical Medicine and International Health 7, 767-774. doi: 935 [pii].

Ramaiah, K. D. and Vijay Kumar, K. N. (2000). Effect of lymphatic filariasis on school children. Acta Tropica 76, 197-199. doi: S0001-706X(00) 00102-9 [pii].

Rahmah, N., Shenoy, R. K., Nutman, T. B., Weiss, N., Gilmour, K. Maizels, R. M., Yazdanbakhsh, M. and Sartono, E. (2003). Multicentre laboratory evaluation of Brugia Rapid dipstick test for detection of brugian filariasis. Tropical Medicine and International Health 8, 895-900. doi: 1102 [pii].

Rahmah, N., Taniawati, S., Shenoy, R.K., Lim, B. H., Kumaraswami, V., Anuar, A. K., Hakim, S. L., Hayati, M. I., Chan, B. T., Suharni, M. and Ramachandran, C. P. (2001). Specificity and sensitivity of a rapid dipstick test (Brugia Rapid) in the detection of Brugia malayi infection. Transactions of Royal Society of Tropical Medicine and Hygiene 95, 601-604.

Seim, A. R., Dreyer, G. and Addiss, D. G. (1999). Controlling morbidity and interrupting transmission: twin pillars of lymphatic filariasis elimination. Revista da Sociedade Brasileria de Medicina Tropical 32 325-328.

Shenoy, R. K. (2006). Lymphatic filariasis in children. Fournal of Communicable Diseases 38, 118-123.

Shenoy, R. K., John, A., Hameed, S., Suma, T. K. and Kumaraswami, V. (2000). Apparent failure of ultrasonography to detect adult worms of Brugia malayi. Annals of Tropical Medicine and Parasitology 94, 77-82.

Shenoy, R. K., Kumaraswami, V., Suma, T.K., Rajan, K. and Radhakuttyamma, G. (1999). A double-blind, placebo-controlled study of the efficacy of oral penicillin, diethylcarbamazine or local treatment of the affected limb in preventing acute adenolymphangitis in lymphoedema caused by brugian filariasis. Annals of Tropical Medicine and Parasitology 93 367-377.

Shenoy, R. K., Suma, T.K., Kumaraswami, V., Padma, S. Rahmah, N., Abhilash, G. and Ramesh, C. (2007a). Dopple ultrasonography reveals adult-worm nests in the lymph vessels of children with brugian filariasis. Annals of Tropical Medicine and Parasitology 101 173-180. doi: 10.1179/136485907X154566.

Shenoy, R. K., Suma, T.K., Kumaraswami, V., Rahmah, N., Dhananjayan, G., Padma, S., Abhilash, G. and Ramesh, C. (2007b) Preliminary findings from a cross-sectional study on lymphatic filariasis in children, in an area of India endemic for Brugia malayi infection. Annals of Tropical Medicine and Parasitology 101, 205-213. doi: 10.1179/ $136485907 X 154548$

Shenoy, R. K., Suma, T. K., Rajan, K. and Kumaraswami, V. (1998) Prevention of acute adenolymphangitis in brugian filariasis: comparison of the efficacy of ivermectin and diethylcarbamazine, each combined with local treatment of the affected limb. Annals of Tropical Medicine and Parasitology 92, 587-594.

Shenoy, R. K., Suma, T. K., Kumaraswami, V., Dhananjayan, G., Rahmah, N., Abhilash, G. and Ramesh, C. (2008). Lymphoscintigraphic evidence of lymph vessel dilation in the limbs of children with Brugia malayi infection. Fournal of Communicable Diseases 40, 91-100.

Shenoy, R. K., Suma, T. K., Kumaraswami, V., Rahmah, N. Dhananjayan, G. and Padma, S. (2009). Antifilarial drugs, in the doses employed in mass drug administrations by the Global Programme to Eliminate Lymphatic Filariasis, reverse lymphatic pathology in children with Brugia malayi infection. Annals of Tropical Medicine and Parasitology 103, 235-247. doi: 10.1179/136485909X398249.

Stanton, A. W., Badger, C. and Sitzia, J. (2000). Non-invasive assessment of the lymphedematous limb. Lymphology 33, 122-135.

Stephenson, L. S., Latham, M. C., Adams, E. J., Kinoti, S. N. and Pertet, A. (1993). Physical fitness, growth and appetite of Kenyan school boys with hookworm, Trichuris trichiura and Ascaris lumbricoides infections are improved four months after a single dose of albendazole. Fournal of Nutrition 123, 1036-1046

Suma, T. K., Shenoy, R. K. and Kumaraswami, V. (2002). Efficacy and sustainability of a footcare programme in preventing acute attacks of adenolymphangitis in Brugian filariasis. Tropical Medicine and International health 7, 763-766. doi: 914 [pii].

Sunish, I.P., Rajendran, R., Mani, T.R., Munirathinam, A. Tewari, S. C., Hiriyan, J., Gajanana, A. and Satyanarayana, K. (2002). Resurgence in filarial transmission after withdrawal of mass drug administration and the relationship between antigenaemia and microfilaraemia - a longitudinal study. Tropical Medicine and International Health 7, 59-69. doi:_828 [pii].

Taylor, M. J., Hoerauf, A. and Bockarie, M. (2010). Lymphatic filariasis and onchocerciasis. Lancet 376, 1175-1185. doi: S0140-6736(10)60586-7 [pii] 10.1016/S0140-6736(10)60586-7.

Vanamail, P., Ramaiah, K. D., Krishnamoorthy, K., Pani, S. P. and Das, P. K. (1992). Distribution oif microfilaria carriers and clinical cases of bancroftian filariasis in relation to family size in an urban situation. Tropical Biomedicine 9, 91-98.

Vanamail, P., Subramanian, S., Das, P. K., Pani, S. P. and Bundy, D. A. P. (1989). Familial clusttering in Wuchereria bancrofti infection. Tropical Biomedicine 6, 67-71.

Weil, G. J. and Ramzy, R. M. (2007). Diagnostic tools for filariasis elimination programs. Trends in Parasitology 23, 78-82. doi: S1471-4922 (06)00293-5 [pii] 10.1016/j.pt.2006.12.001.

World Health Organization (2000). Preparing and implementing a national plan to eliminate lymphatic filariasis. WHO/CDS/CPE/CEE/ $2000.15,29 \mathrm{pp}$

World Health Organization (2010a). Global programme to eliminate lymphatic filariasis. Weekly Epidemiological Record 85, 365-372.

World Health Organization $(2010 b)$. Working to overcome the global impact of neglected tropical diseases. First WHO Report on Neglected Tropical Diseases, WHO/HTM/NTD/2010.1, 172 pp.

World Health Organization $(2010 c)$. World Health Organization Global Programme to Eliminate Lymphatic Filariasis: Progress report 2000-2009 and strategic plan 2010-2020 of the global programme to eliminate lymphatic filariasis: halfway towards eliminating lymphatic filariasis. WHO/HTM/NTD/PCT/2010.6, 79 pp.

Witt, C. and Ottesen, E.A. (2001) Lymphatic filariasis: an infection of childhood. Tropical Medicine and International Health 6, 582-606. doi: tmi765 [pii].

Witte, M.H., Jamal, S., Williams, W. H., Witte, C. L. Kumaraswami, V., McNeill, G. C., Case, T. C. and Panicker, T. M. (1993). Lymphatic abnormalities in human filariasis as depicted by lymphangioscintigraphy. Archives of Internal Medicine 153, 737-744. 